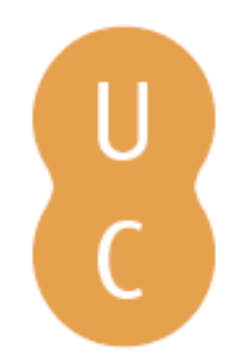

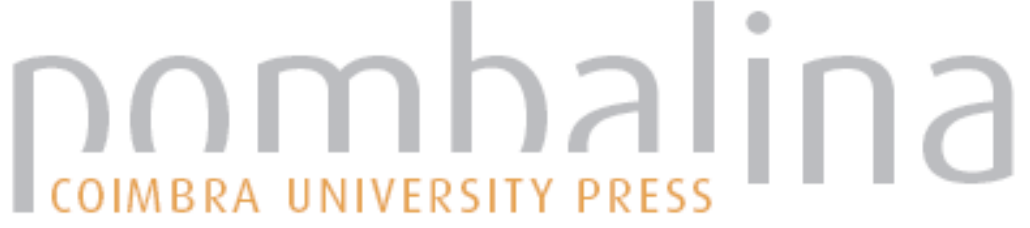

\section{Le novelle petroniane: forme di riscrittila dei modelli}
Autor(es):
Dimundo, Rosalba
Publicado por: Centro de Estudos Clássicos e Humanísticos
URL persistente:
URI:http://hdl.handle.net/10316.2/39252
DOI:
DOI:http://dx.doi.org/10.14195/978-989-26-1229-4_10

Accessed : $\quad$ 26-Apr-2023 14:14:00

A navegação consulta e descarregamento dos títulos inseridos nas Bibliotecas Digitais UC Digitalis, UC Pombalina e UC Impactum, pressupõem a aceitação plena e sem reservas dos Termos e Condições de Uso destas Bibliotecas Digitais, disponíveis em https://digitalis.uc.pt/pt-pt/termos.

Conforme exposto nos referidos Termos e Condições de Uso, o descarregamento de títulos de acesso restrito requer uma licença válida de autorização devendo o utilizador aceder ao(s) documento(s) a partir de um endereço de IP da instituição detentora da supramencionada licença.

Ao utilizador é apenas permitido o descarregamento para uso pessoal, pelo que o emprego do(s) título(s) descarregado(s) para outro fim, designadamente comercial, carece de autorização do respetivo autor ou editor da obra.

Na medida em que todas as obras da UC Digitalis se encontram protegidas pelo Código do Direito de Autor e Direitos Conexos e demais legislação aplicável, toda a cópia, parcial ou total, deste documento, nos casos em que é legalmente admitida, deverá conter ou fazer-se acompanhar por este aviso.

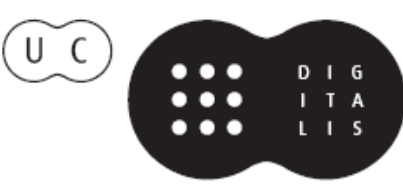


FRANCISCO DE OLIVEIRA

PAOLO FEDELI

DELFIM LEÃO

Coordenadores

\section{- ROMANCE ANTIGO ORIGENS DE UM GÉNERO LITERÁRIO}

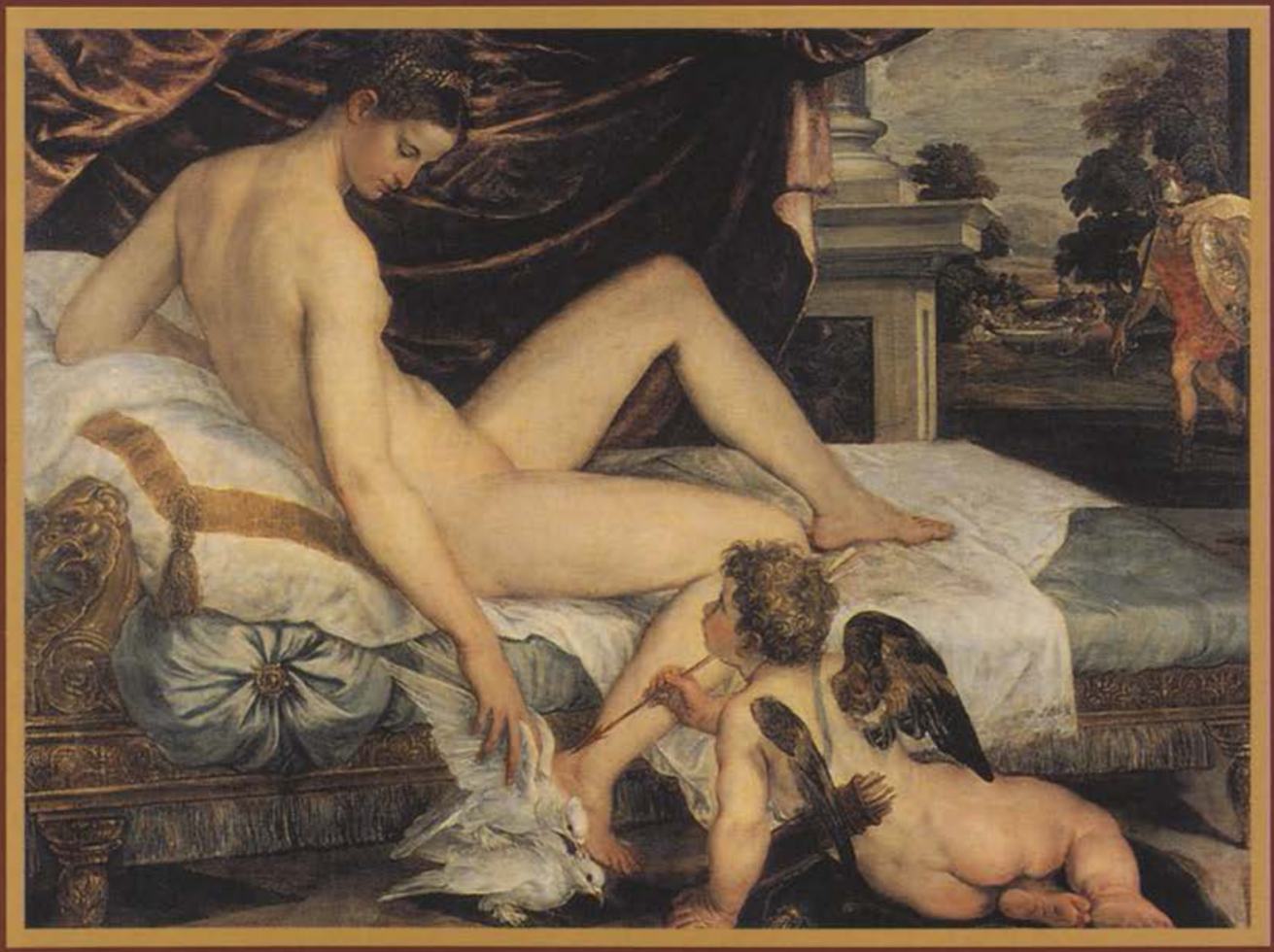

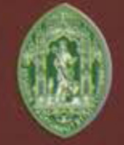

Universidade de Coimbra

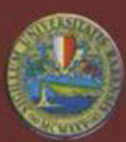

Università degli Studi di Bari

COIMBRA

2005 


\title{
LE NOVELLE PETRONIANE: FORME DI RISCRITTURA DEI MODELLI
}

\author{
ROSALBA DIMUNDO \\ Universidade de Bari
}

1. Gli incerti contorni del Satyricon - opera che, com'è noto, viola le categorie tradizionali di generi classici - sembrano riflessi anche nel singolare atteggiamento di Petronio nei confronti dei modelli di volta in volta impiegati, al punto che spesso, accanto alle fonti principali, non è raro individuare una molteplicità di apporti dei generi letterari e degli autori più diversi: il modello adottato, cosi, non condiziona prepotentemente la narrazione, ma all'interno di essa subisce una radicale metamorfosi.

Il senso di tale operazione risulterà più chiaro se rivolgiamo la nostra attenzione alle novelle: momenti ben definiti della diegesi quali sono, infatti, esse ci permettono di apprezzare fino in fondo la riscrittura petroniana dei modelli che di volta in volta vengono adottati e resi chiari ai lettori. Attraverso elementi caratterizzanti della narrazione, quali lo statuto del narratore o le poliedriche figure dei personaggi, infatti, l'autore, più o meno nascosto, 'opera in modo tale da riformulare decisamente la sua fonte.

2. Nel caso delle novelle principali, quelle cioè narrate da Eumolpo in momenti diversi del romanzo, la singolare opera di degradazione dei modelli assume proporzioni quasi eclatanti: la prima, quella ambientata a Pergamo, com'è noto, rinvia ad un contesto del Simposio platonico (174a-174d). La coppia puer/Eumolpo, infatti, rivela una marcata analogia con la coppia Alcibiade/Socrate, che in quei capitoli dell'opera platonica era al centro dell'attenzione e dell'interesse generale. In

1 Il riferimento è qui al saggio, denso di suggestioni, di G.B. Conte, L autore nascosto. Un'interpretazione del 'Satyricon', Bologna 1997 (trad. di The Hidden Author: An Interpretation of Petronius' 'Satyricon', Berkeley 1996. 
entrambi i casi troviamo un giovanetto (puer/ Alcibiade) e un pedagogo-filosofo (Eumolpo/Socrate), i quali si confrontano sulla tematica del rapporto affettivo tra maestro e allievo. Nel Simposio, tuttavia, è Alcibiade a tentare di sedurre il proprio maestro, mentre nella novella petroniana è il pedagogo ad attentare alla pudicizia del suo allievo. Da questa mutata prospettiva ben si comprende perché Eumolpo dica che la madre del puer lo considera come il filosofo per eccellenza $(85,2$ tamquam unum ex philosophis): era questa, infatti, la definizione che si dava di Socrate. Tuttavia nel contesto platonico, che rivela altre sorprendenti convergenze con il racconto petroniano, ${ }^{2}$ la seduzione non si verifica, al contrario della novella in cui, a seconda delle circostanze, seduttore è l'uno o l'altro dei protagonisti. Alla fine del racconto, se pure Eumolpo tornerà ad assumere il ruolo di austero filosofo, indossando di nuovo i panni di novello Socrate, egli diventerà casto non per un convinto desiderio di continenza, ma per comprensibile sfinimento, dopo le reiterate prestazioni sessuali. Il rovesciamento dello schema platonico coinvolge in primo luogo il protagonista del racconto: colui che nelle fasi iniziali della novella era stato seduttore, viene a sua volta sedotto e diviene, quindi, casto proprio quando si riappropria del nobile modello socratico, rispetto al quale Petronio realizza, dunque, un'accorta opera di demistificazione.

La geniale originalità petroniana, poi, va rintracciata anche nella riformulazione dello statuto del narratore, che si distingue per una sostanziale novità rispetto al modello. Nella novella dell'efebo il narratore, che è anche personaggio della vicenda narrata, adotta la tecnica della narrazione in prima persona ${ }^{3}$ e in questo suo assurgere a protagonista del fatto narrato vi è un chiaro segnale al lettore, il quale deve intuire che nell'ambito del romanzo in primo luogo Eumolpo si accinge a sottrarre ad Ascilto la funzione deuteragonistica, in secondo luogo che egli concorrerà d'ora in poi a far progredire la narrazione negli inevitabili momenti di stasi. Esiste, dunque, un legame profondo tra narrazione principale e novella, che si traduce in una precisa funzione didattica: Eumolpo, infatti, oltre a consolare Encolpio,

2 Sul motivo della degradazione del modello platonico cf. R. Dimundo, $D a$ Socrate a Eumolpo. Degradazione dei personaggi e delle funzioni nella novella del fanciullo di Pergamo, MD 10-11,1983, 255-265 e G. Sommariva, Eumolpo, um "Socrate epicureo" nel'Satyricon', ASNP 14,1984, 25-58.

${ }^{3}$ Il suo è lo statuto tipico, per riprendere la nota formulazione di Genette, del narratore intradiegetico-omodiegetico: cf. G. Genette, Figure III. Discorso del racconto, trad. it., Torino 1976, 296. Si leggano, poi, le sue puntualizzazioni in Nuovo discorso del racconto, trad. it., Torino 1987, 98 sgg. 
duramente provato dal tradimento di Gitone, riesce a dimostrargli che tutti gli efebi sono incostanti e volubili; un'analoga funzione nel racconto hanno, del resto, i discorsi sviluppati di volta in volta dai diversi interlocutori dell'opera platonica, punto di partenza dell'elaborazione petroniana.

La narrazione in prima persona, poi, concorre a rimarcare ulteriormente la distanza dalla fonte e a mettere in luce l'elaborazione petroniana anche attraverso il ricorso all'intersezione di generi e modelli. E' noto che la narrazione in prima persona rappresenta un espediente per conferire maggiore veridicità al racconto, proprio perché il narratore si fa protagonista dei fatti narrati e rinvia probabilmente a tecnica della milesia anche la finzione, in base alla quale il narratore principale del romanzo affida ad altra persona (in questo caso ad Eumolpo) il racconto di una singolare vicenda, che in tal modo acquista maggiore credibilità. Attraverso il suo racconto, poi, il narratore dà luogo a un complesso sistema relazionale con quanti ascoltano o leggono la sua novella. Un primo rapporto, infatti, è quello che si crea fra il narratore e il destinatario, intendendo in tal caso per destinatario l'interlocutore che si colloca nella cornice del racconto, funge da pubblico per il narratore e, di conseguenza, diventa il primo bersaglio dei suoi artifici retorici.

Nel caso delle novelle petroniane il narratario può essere singolo (Encolpio nella novella dell'efebo) oppure può essere rappresentato da un gruppo di persone (i commensali nel caso delle novelle di Nicerote e Trimalchione, i naviganti nella novella della matrona): si tratterà, comunque, di narratari consenzienti, ${ }^{4}$ la cui fiducia dovrà essere catturata in vario modo. La novella dell'efebo costituisce l'unico caso in cui il narratario non conosce affatto il narratore: a Encolpio il vecchio lacero e trasandato che lo interpella nella pinacoteca è totalmente ignoto, diversamente da quanto avviene nel contesto platonico, in cui la familiarità che lega reciprocamente gli interlocutori è concretamene testimoniata dal contesto simposiale che fa da cornice; nella rielaborazione che del contesto platonico fa Petronio, allora, è indispensabile che Eumolpo avvalori il suo racconto rendendosi protagonista degli eventi narrati. Il narratario rimarrà colpito e

${ }^{4}$ Come sottolinea S. Chatman (Storia e discorso. La struttura narrativa nel romanzo e nel film, trad. it., Parma 1981, 284) «un narratario consenziente può mostrare che gli sforzi del narratore diretti a convincere, a imporre la sua versione dei fatti raggiungono lo scopo [...]. Nel più semplice dei casi, dove non c'è motivo di dubbio, l'accettazione del narratario garantisce l'attendibilità del narratore»; cf. anche L. Cicu, La matrona di Efeso in Petronio, SIFC 4,1986, 270. 
influenzato dal racconto a lui solo diretto, al punto da subire una profonda trasformazione del suo stato d'animo.

All'articolazione del racconto dell'avventura pergamena di Eumolpo in sezioni ben distinte fa riscontro un'analoga suddivisione sul piano formale, a fronte di una evidente uniformità diegetica $\mathrm{e}$ stilistica del modello codice. Nell'esordio della novella il narratore/protagonista si esprime con grande accuratezza, costretto com'è a giustificare opportunamente la sua presenza in quell'hospitium in qualità di pedagogo. Dopo la definizione della situazione di base, Petronio ricorre ad una sospensione del ritmo narrativo $(85,3)$, che serve ad accrescere l'attesa e l'attenzione dei destinatari. Il pedagogo, che è riuscito a creare l'atmosfera adatta ai suoi tentativi, è pronto al primo approccio col puer $(85,5)$ e per non fallire subito in modo clamoroso deve necessariamente far ricorso alla propria abilità non solo di seduttore ma anche di oratore: l'atmosfera di segreta intimità, rotta solo dal timidissimum murmur, sottolinea la delicatezza del momento. Nel prosieguo della narrazione, visti i risultati positivi del primo votum, Eumolpo ha ormai la strada libera per la seconda e, poi, per la terza e più audace richiesta. Quest'ultima è strettamente collegata con la prima, sebbene a differenziare i due vota intervenga un'amplificazione lessicale (la maggiore complessità nella formulazione del terzo votum) e psicologica (l'atteggiamento indiscreto di Eumolpo). Dopo la conclusiva esibizione erotica del pedagogo, che però ha provocato la sdegnata reazione del puer non esaudendo il terzo votum, la situazione è giunta a un punto di stasi. Sarà ancora Eumolpo a rimettere in moto l'azione, nell'intento di saggiare nuovamente la disponibilità erotica del puer. Nel capitolo finale della novella la narrazione ha un ritmo più rapido, anche se mancano passaggi repentini. Il cambiamento è determinato dalla diversa psicologia dei personaggi principali: d'ora in poi, infatti, essi agiranno su un piano di assoluta sincerità e metteranno da parte le motivazioni sinora fittizie delle loro azioni. Protagonista indiscusso, nella seconda parte della novella, è il puer. Solo al termine della narrazione, analogamente al ruolo assunto all'inizio, Eumolpo torna a rivestirsi dei panni di pedagogo: lo sottolinea il conclusivo plane vehementer excandui $(87,9)$, che si ricollega al tam vehementer excandui dell'esordio $(85,2)$.

3. Lo stile delle novelle di Eumolpo si adatta di volta in volta agli eventi narrati. Nel racconto del puer, sebbene egli assuma, a seconda delle esigenze, ora il ruolo di austero pedagogo, ora quello di amator, in entrambe le situazioni la narrazione procede lungo i binari di un 
vigile controllo stilistico, imposto ad Eumolpo, dal destinatario (Encolpio), certamente non incolto se frequenta una pinacoteca. Nella novella ambientata ad Efeso, né l'ambiente (la nave di Lica, che è stata poco prima teatro di una colossale zuffa) né i narratari (oltre a Encolpio e Gitone, i rozzi naviganti e l'altrettanto rozzo nocchiero, la dissoluta Trifena) sono tali da imporgli un'analoga scelta stilistica. Tuttavia il vecchio poeta ha un suo stile, che mantiene inalterato in tutto il romanzo, sebbene venga adattato in maniera perfetta ai personaggi e alle situazioni: si servirà a piene mani del linguaggio militare per descrivere i reiterati e tenaci assalti del miles alla pudicizia della matrona, accompagnerà con altisonanti citazioni dall'epos le magniloquenti esortazioni dell'ancella, affiderà ai toni enfatici e solenni della tragedia il compito di sottolineare i progressivi cedimenti della vedova; anche in questo caso manterrà ad un livello elevato persino la descrizione dell'unione sessuale del soldato e della matrona. Le riflessioni sul linguaggio di Eumolpo, che si configura come il perfetto riflesso del suo grado culturale, ci permettono, dunque, si indirizzare l'analisi all'altra grande novella del Satyricon. La struttura di fondo della novella della matrona di Efeso è presente già nella raccolta esopica $(109$ Halm $=299$ Hausrath $)$ e, nonostante lo schema più semplice a due personaggi, esistono peculiari coincidenze nello schema narrativo e nella tecnica del racconto. Il più complesso intreccio a tre personaggi compare in Fedro (App.13) e godrà di una notevole fortuna nella favolistica delle culture più diverse. Tuttavia se la fonte diretta di Petronio sembrerebbe essere quella fedriana, il suo modello-codice è costituito dall'Eneide di Virgilio e Petronio si preoccupa costantemente di fornire al lettore gli strumenti che lo mettano in grado di decodificare il testo, come dimostrano le nobili citazioni virgiliane fatte dall'ancella nelle sue perorazioni. ${ }^{5}$ Alla luce di tale modello, allora, è possibile considerare in modo diverso gli stessi protagonisti, che costituiscono tutti la riproduzione degradata di celebri personaggi virgiliani: se la vedova inizialmente inconsolabile e succes-sivamente facile preda della passione d'amore rappresenta una chiara ripresa con degradazione della Didone virgiliana, l'ancella, sua provvida consigliera, prende il posto della sorella Anna - che

${ }^{5}$ Il riferimento al contesto virgiliano, del resto, è suggerito già nella cornice iniziale della novella, quando Eumolpo si accinge a narrare l'episodio e l'attenzione dei presenti è concentrata sulla figura del vecchio poeta: 110,8 conversis igitur omnium in se vultibus auribusque orsus est; tali parole riecheggiano, infatti, l'esordio del racconto di Enea a Didone (Aen. 2, 1-2 conticuere omnes intentique ora tenebant; / inde toro pater Aeneas sic orsus ab alto). 
nell'Eneide svolge un'analoga funzione - e ad Enea corrisponde l'umile soldato. Come nel modello epico l'eroe si dimostra felicemente facundus, narrando tutto d'un fiato le sue peripezie nel secondo libro dell'Eneide, analogamente le stesse qualità oratorie caratterizzano il miles nella novella petroniana. Enea, inoltre, finisce per rimpiazzare il morto Sicheo, come accade nel Satyricon al soldato che diventa il provvidenziale sostituto del defunto marito della matrona. Petronio, tuttavia, rovescia il suo modello: mentre, infatti, nell'Eneide è Didone a soccorrere Enea, nella novella è il soldato che esorta la donna, con offerte di cibo e di vino, a reviviscere.

Un analogo processo di degradazione coinvolge il luogo in cui è ambientato il racconto: la fastosa cornice della reggia nel testo epico è stata sostituita addirittura da un sepolcro. La conclusione della vicenda erotica in Petronio, però, è del tutto diversa da quella virgiliana. La storia d'amore di Didone ed Enea è ostacolata, infatti, dalle convenzioni e dalle regole di comportamento che impongono a Didone di restare fedele al ricordo del marito, sebbene l'eroina virgiliana, analogamente al personaggio petroniano, riesca a superare con relativa facilità un tale divieto; invano si troverà a combattere, invece, contro il destino stesso di Enea, che non consentirà all'eroe peregrinante di fissare la sua dimora a Cartagine. La storia d'amore tra Enea e Didone, pertanto, è destinata ineluttabilmente al fallimento e alla tragica conclusione che vedrà il suicidio della regina. Nel racconto petroniano, invece, esiste solo un antagonista: il corpus iacentis del marito della matrona; si tratta, dunque, di un antagonista talmente innocuo, che finirà per trasformarsi in aiutante quando sostituirà il corpo trafugato di uno dei latrones. Per di più, la matrona petroniana esce riscattata anche dal biasimo nei confronti dei suoi mutati costumi morali, perché gli Efesini non potranno condannare il comportamento della vedova, destinata a rimanere un luminoso esempio per una città che di un exemplum ha bisogno, ma dovranno limitarsi a chiedersi, con stupore ed ammirazione al tempo stesso, qua ratione mortuns isset in crucem $(112,8)$. Ad Efeso la donna - che costituiva l'esempio più grande di pudicizia - potrà continuare a fregiarsi del lusinghiero appellativo di pudicissima anche quando sarà divenuta un modello insigne d'impudicizia.

Un'ulteriore differenziazione rispetto al modello emerge dall'analisi della funzione del narratario. Se, infatti, Enea non è noto ai suoi interlocutori diretti e sulle prime si mostra quasi reticente nei confronti dell'invito rivoltogli dalla regina Didone a raccontare le sue tristi vicende della caduta di Troia e delle sue peregrinazioni (il racconto, 
infatti, gli procura un infandus dolor: Aen. 2,3), nel caso della novella della matrona, Eumolpo non solo è noto al suo pubblico, ma ha acquistato singolari benemerenze riportando la pace nella turbolenta congrega: forte di questo vantaggio egli può tranquillamente narrare, sicuro d'essere creduto, una storia di cui non è stato né protagonista né testimone. Coinvolgerà a tal punto i suoi ascoltatori che, quando nella conclusione della novella verrà dipinto lo stupore del populus per la straordinaria ascesa del corpus iacentis sulla croce, tale meraviglia si rivelerà quanto mai comica per i narratari, ben consapevoli del motivo reale. Se nel racconto di Enea, poi, non c'è mai interruzione del discorso del narratore attraverso l'apostrofe agli ascoltatori, nella novella raccontata da Eumolpo è possibile rintracciare tre momenti in cui il narratore interviene in prima persona sul piano principale della narrazione: nel primo caso $(111,13$ nemo invitus audit cum cogitur aut cibum sumere aut vivere) la massima di carattere universale ha il fine anche di saggiare e stimolare i narratari, mentre in altri due luoghi del racconto diverse sono le finalità del narratore. A 112,1 (ceterum scitis quid plerumque soleat temptare humanam satietatem) l'intervento di Eumolpo si configura come un commento dell'avvenimento raccontato, contribuisce ad evidenziarne l'importanza e a richiamare l'attenzione sull'evento ancor più significativo che sta per prodursi: siamo, insomma, nella fase cruciale della vicenda, che a partire da quel momento assumerà esiti nuovi. A 112, 2, il quid diutius moror? è una pseudo-domanda che il narratore non rivolge a se stesso ma al narratario, ${ }^{6}$ con il fine di spiegare il sorprendente cedimento sessuale della matrona, che si verificherà subito dopo.

4. Anche il racconto di Nicerote persegue intenti di demistificazione dell'Eneide: l'emistichio virgiliano haec ubi dicta dedit (61,5), che segna il passaggio di Nicerote dalla perplessità alla decisione di narrare la sua sconvolgente esperienza e rende immediatamente chiaro al lettore l'ipotesto, trasforma il narratore in una grottesca caricatura dell'Enea virgiliano, attraverso una composita trama referenziale che recupera elementi del IV e del VI libro dell'Eneide; come Enea, anche Nicerote ha la sua Didone, un'ostessa dai facili costumi che è vedova e si chiama Melissa (quasi a ricordare Elissa, appellativo della regina di Cartagine). Il cammino notturno irto di pericoli che Nicerote deve percorrere per raggiungere la sua vedova, inoltre, costituisce la ripresa in chiave parodica della catabasi di Enea nel VI dell'Eneide: entrambi i

\footnotetext{
${ }^{6}$ Per tale risorsa retorica cf. G. Prince, Narratologia, trad. it., Parma, 1984, 29.
} 
protagonisti, infatti, si avventurano nel regno dei morti (cimitero/ inferi); tuttavia, se Enea lo fa nella piena coscienza della protezione divina e della propria missione, Nicerote viene attratto suo malgrado in quel mondo tenebroso. Alla pavida incoscienza di Nicerote, che si ferma nel contesto statico di un cimitero, in cui può avere un contatto diretto solo con stelae e monimenta, corrisponde, però, la cosciente azione di Enea, il quale, dopo un iniziale smarrimento, prende a narrare le proprie vicende alle ombre che gli si affollano intorno. E, infine, il tentativo di Nicerote di farsi largo fra quelle inconsistenti e misteriose figure della notte, assestando fendenti a destra e a manca, costituisce una parodica ripresa del proposito iniziale di Enea di difendersi dai mostri che custodiscono la porta del regno di Dite. Risultano evidenti, allora, i solidi agganci tra il romanzo e l'epos, sebbene proprio lo scarto dalla poesia epica contribuirà a mettere in luce la caratteristica principale: l'ironia, infatti, scaturisce dalla degradazione del modello, che dovrà essere seguito fino ad un immancabile, parodico rovesciamento conclusivo.

Nel caso della novella del vetro infrangibile, il discorso sulle fonti e sulla loro degradazione nel Satyricon acquista contorni diversi e non può essere scisso da alcune considerazioni sul rapporto fra l'autore e la realtà socioeconomica che in questa novella è possibile cogliere: non è difficile, infatti, scorgere tra le righe sia l'interesse di Petronio per problemi di vitale importanza al tempo suo, sia il suo sguardo attento anche ai progressi tecnologici degli anni più recenti. Plinio il Vecchio, in cui si rintraccia la stessa vicenda, ${ }^{7}$ descrive con estrema concisione senza peraltro nascondere il proprio scetticismo - il fatto privo del cruento epilogo, mentre l'artefice viene mandato a morte nel racconto di Cassio Dione $(57,21,5-7)$, che rappresenta un'amplificazione probabilmente fantasiosa alla luce sia del sintetico passo pliniano, sia anche del più complesso e vivace contesto petroniano; ${ }^{8}$ in quest'ultimo,

${ }^{7}$ Cf. Nat. 36,195 ferunt Tiberio principe excogitato vitri temperamento, ut flexile esset, totam officinam artificis eius abolitam, ne aeris, argenti, auri metallis pretia detraherentur, eaque forma crebrior diu quam certior fuit. La stessa vicenda ricomparirà nei testi medievali, ampiamente basati sul testo petroniano, di Isidoro di Siviglia (Etym.16,16,6) e di Giovanni di Salisbury (Policr. 4,5,521b-d).

${ }^{8}$ Secondo Cassio Dione, infatti, la gelosia di Tiberio nei confronti dell'artefice aveva un precedente, e già allora la posizione del principe era stata improntata a durezza dettata dalla gelosia: l'architetto, ricompensato col denaro per aver facilmente e mirabilmente restaurato un portico ormai in sfacelo, era stato però cacciato da Roma. L'episodio del vetro si situa, secondo Cassio Dione, al momento del ritorno 
accanto a una peculiare attenzione alla struttura del racconto e al suo risvolto sociale, è possibile apprezzare un ritmo rapido e incalzante, che sfocia nella conclusione inattesa. Il racconto, poi, è costruito sull'opposizione caratteriale dei due personaggi, in cui l'ingegno del faber trova un imprevisto ostacolo nell'astuzia calcolatrice dell'imperator e deve necessariamente soccombere. Ma Petronio non nasconde l'insegnamento che dalla vicenda si può trarre: nella conclusione della novella l'artefice, di cui all'inizio era stata descritta la legittima felicità per la sua straordinaria scoperta, viene privato, oltre che della vita, anche del sogno d'aver ottenuto il successo grazie al proprio ingenium: gli si sottrae, così, com'è stato giustamente osservato, «la possibilità di incutere in qualche modo paura a chi detiene il potere, di mettere in crisi gli equilibri costituiti»?

La funzione didattica della novella, allora, emerge proprio dalle osservazioni conclusive del narratore: le leggi economiche, cioè, sono troppo importanti e sui potenti incombono troppo grandi responsabilità, perché si debba tener conto della vita di un faber. Per Petronio «era indiscutibile che l'oro dovesse conservare la sua funzione di metallo privilegiato, fondamentale punto di riferimento simbolico per le valutazioni e gli scambi, perché le innovazioni in questo campo avrebbero comportato il crollo di un sistema economico e quindi sociale». ${ }^{10}$

dell'artefice a Roma per chiedere perdono al principe, ma nella conclusione della vicenda risultano ambigue le ragioni della vendetta del principe: il faber, infatti, «accostatosi all'imperatore in atteggiamento da supplice, nello stesso tempo scagliò volontariamente al suolo una coppa di vetro; qua e là questa era ammaccata e contusa; ma, dopo che la strofinò con le sue mani, essa ritornò subito intatta; in tal modo pensava di ottenere il perdono, ma l'imperatore lo mandò a morte».

${ }^{9}$ Cf. G. Polara, La tradizione medievale della novella petroniana del vetro infrangibile, in Semiotica della novella latina, cit., 131-42.

${ }^{10} \mathrm{Cf}$. Polara, cit., 141-42. Forse proprio muovendo da tali constatazioni, relative alla novella del vetro infrangibile, è possibile individuare i limiti dell'analisi che del realismo petroniano ha fatto Erich Auerbach. Si sa bene che egli, nel capitolo intitolato Fortunata della sua opera più nota Mimesis. Il realismo nella letteratura occidentale, trad. it., Torino $1956,30-57$ ha posto a confronto il realismo petroniano con l'atteggiamento della mentalità arcaica (Omero, i tragici) e ha creduto di poter individuare non solo le novità, ma anche i limiti della posizione assunta da Petronio. Rovesciando il ben noto giudizio di Auerbach, secondo cui Petronio è del tutto indifferente ai problemi socio-economici della società in cui vive e produce i suoi documenti di letteratura, appare legittimo sostenere, invece, che il realismo di Petronio, se si considera la sua presentazione dei fatti socioeconomici, rivela una carica dinamica molto più forte di quanto Auerbach abbia scorto nel Satyricon e una presa di coscienza problematica non solo dell'emergere di un nuovo ceto, ma anche dei 
5. Nel rapporto con i narratari, Nicerote e Trimalchione - che, noti come sono solo a una parte di essi, partono da una posizione di svantaggio rispetto al narratore Eumolpo - dovranno comportarsi in modo da conquistarsi la loro fiducia con ogni stratagemma. Tuttavia, se Trimalchione nella novella del vetro infrangibile non deve penare troppo per stabilire un rapporto di fiducia con i suoi narratari (dietro di sé ha una storia con una sua tradizione -quella che riferisce l'accaduto a Tiberio- di cui può farsi forte e, per di più, egli sa sfruttare la presenza del personaggio paradigmatico quale l'imperatore), narrare novelle di streghe e licantropi comporta ben altri problemi: non stupisce, quindi, che i narratori adottino tutt'altra strategia nei confronti dei narratari: la loro risorsa maggiore sarà una continua proclamazione della verità. In entrambi i casi, poi, i narratori sono consapevoli di raccontare eventi talmente straordinari che ben difficilmente potranno essere creduti: di conseguenza tali avvenimenti dovranno essere esposti con chiarezza, ricorrendo magari alla ripetizione. In racconti dominati da suspense, del resto, i due narratori sfruttano la superiorità che deriva dall'essere stati testimoni e protagonisti della vicenda e permette loro di orientare la narrazione come meglio desiderano, mettendo in evidenza i momenti secondo loro più importanti, comunicando continuamente ai narratari le loro impressioni sui fatti raccontati e coinvolgendoli sempre più in quelle tenebrose vicende. Nella conclusione delle novelle l'opera di convincimento dei pur rozzi narratori avrà, dunque, raggiunto i suoi effetti immediati, se i narratari saranno sinceramente convinti di aver ascoltato il racconto di fatti realmente accaduti e provvederanno agli scongiuri di rito.

Sul piano formale le novelle di Nicerote e di Trimalchione si distinguono per una netta differenza nei confronti dello stile dello scholasticus Encolpio, spesso vivacemente espressivo, ma sempre raffinato. Il liberto Trimalchione e, accanto a lui, il liberto Nicerote non riescono a celare la loro provenienza sociale: la liberazione dalla schiavitù e l'acquisita indipendenza economica, infatti, non hanno potuto garantire il possesso dell'unico bene che i veri discendenti degli antichi ottimati, gli ingenui per nascita, possono ancora orgogliosamente rivendicare come esclusivo: la cultura, l'istruzione e dunque la capacità di esprimersi in uno stile accurato, che segna la loro presunta e illusoria superiorità nei confronti del ceto emergente. Nel caso delle

profondi cambiamenti in campo sociale ed economico che a tale mutata situazione erano inevitabilmente legati. 
storie di streghe e fantasmi, che afferiscono a un filone narrativo popolare e mai potrebbero essere narrate da uno scholasticus o da un dotto poeta, è lo stesso status sociale dei narratori a funzionare «da ironica presa di distanza».

Nei racconti di Trimalchione si potrà, tutt'al più, notare una differenza nel modo di narrare, che è molto più rapido ed essenziale nella novella del vetro infrangibile, in cui la non partecipazione all'accaduto impone di festinare ad eventum verso la tragica conclusione della vicenda: ma proprio quando la tensione sta per raggiungere il suo culmine, l'immagine dell'artefice che putabat se coleum Iovis tenere $(51,5)$ rende vani gli sforzi di concentrazione stilistica da parte di Trimalchione.

Diversa è la tecnica della novella del licantropo, caratterizzata da lunghi indugi sulle determinazioni ambientali e sulle informazioni relazionali del narratore (la sua condizione sociale; il suo rapporto con Melissa, presentata con il riferimento al luogo d'origine, al nome, al mestiere del marito). In particolare, tale personaggio femminile risulta proiettato su due piani diversi: il primo è quello della realtà (si tratta pur sempre di un'adultera), l'altro è quello dell'apparenza (la sua presentazione come benemoria), che tuttavia non inganna affatto $\mathrm{i}$ destinatari del racconto, ma agli occhi del lettore diviene il personaggio determinante dell'intreccio per due volte: è lei, a causa della morte del marito, ad imporre a Nicerote lo spostamento notturno; è sempre lei a fornire a Nicerote la spiegazione della sua avventura.

Il miles, caratterizzato sin dall'inizio da elementi che preludono alla sua metamorfosi (cfr. 62,2 fortis tamquam Orcus), è un personaggio muto (l'unica sua manifestazione verbale è l'ululato al momento della metamorfosi), che tuttavia supplisce alla mancanza di interventi verbali con azioni stupefacenti. La licantropia, se lo rende temibile a Nicerote, lo fa divenire patetico agli occhi dei destinatari della novella, dal momento che la metamorfosi gli procura solo una serie di danneggiamenti: dapprima la terribile ferita alla gola, poi la degradazione da uomo ad animale (come tale è sempre caratterizzato da Nicerote: cfr., infatti, anche 62,13 tamquam bovis), infine l'esclusione dai consueti rapporti umani.

6. Il racconto di Trimalchione, che si sviluppa sulla scia di quello di Nicerote, tenta di sfruttare accortamente lo stato di meraviglia mista a terrore che si è impadronito dei convitati. Non c'è dunque soluzione di continuità - neanche a livello psicologico - tra la storia di Nicerote e quella di Trimalchione, che va letta alla luce dell'espressione iniziale 
(63,1 attonitis admiratione universis): Trimalchione, infatti, vuole solo stupire i suoi ascoltatori, ma non possiede gli idonei strumenti retorici: egli stesso ne è ben consapevole, tanto che sua unica preoccupazione sarà quella di intervenire, nel corso del racconto, con reiterate assicurazioni di veridicità. Se, però, i narratari restano irretiti dall'atmosfera tenebrosa e dai particolari terrificanti del discorso, il lettore, svincolato com'è dal "tempo del racconto», è in grado di leggere in modo diverso il «tempo della storia». Da questa mutata prospettiva non sarà difficile accorgersi degli elementi di comicità qua e là disseminati: ad esempio nella rappresentazione di Trimalchione giovane capillatus (dato che contrasta con la sua attuale calvizie), o nella descrizione del mastodontico e sciocco Cappadoce, l'unico personaggio della novella. ${ }^{11}$

7. La straordinaria modernità del romanzo petroniano non fu compresa dal pubblico, sia pur raffinato del suo tempo e, insieme con le alterne vicende della tradizione del testo, determinò la sua scarsissima notorietà. Il pregevole tessuto stilistico del Satyricon, l'uso e il riuso della letteratura piegata a diversificate istanze narrative presupponevano un lettore in grado di leggere - attraverso la lente "plurifocale" che l'autore, più o meno scopertamente, gli forniva - un'opera destinata a essere amata solo a distanza di molti secoli. In tale prospettiva esegetica anche le novelle acquistano un significato diverso, che va ben oltre l'aspetto ludico, perché il loro rapporto con le fonti non si limita, come si è visto, ad un infecondo 'maquillage' del modello, ma si qualifica nei termini di una originalissima rilettura. Lungi dal rappresentare paradigmi rigidi e intangibili, icone venerabili

${ }^{11}$ Trimalchione si limita a partecipare alla vicenda e non sottolinea un suo ruolo di rilievo. Dopo una rapida presentazione, che serve ad inquadrare il racconto dal punto di vista temporale anche mediante il riferimento alla sua capigliatura $(63,3 \mathrm{cum}$ adhuc capillatus essem), il protagonista/narratore viene subito riassorbito nella pluralità dei numerosi partecipanti alla veglia funebre $(63,4$ nos plures in tristimonio essemus; 63,5 habebamus Cappadocem; 63,6 audimus gemitum; 63,7 baro ... noster, 63,8 nos redimus); persino la madre è appiattita nella presenza corale di quanti agiscono nel tristimonium, né acquista una sua fisionomia quando si accorge che al posto del figlio c'è un fantoccio di paglia. La figura della madre, d'altronde, ha solo un valore strutturale: essa serve ad aprire e chiudere la vicenda. Protagonista indiscusso è il Cappadoce, descritto come se si trattasse di un eroe (63,5 longus, valde audaculus et qui valebat: poterat bovem iratum tollere). Ma è proprio la figura possente a decretarne la fine: gli attributi morali e materiali (63,6 audacter stricto gladio), invece di consentirgli di aver la meglio sulle streghe, fanno di lui un facile bersaglio e lo predispongono a un destino certo e inesorabile. 
della letteratura, i modelli delle novelle - ma la prospettiva va allargata al romanzo nel suo complesso - divengono con Petronio materia duttile al punto da ricostituirsi in forme nuove. L'assenza di confini definiti, allora, costituisce, in modo apparentemente paradossale, lo straor-dinario pregio di un'opera, che suscita - oggi più di ieri- un inesauribile fascino sul lettore, chiamato a leggerla attivamente, a darle cioè la forma e la voce suggerite di volta in volta dalla sua sensibilità di interprete. 